\title{
The psoas muscle as cause of low back pain in infantile cerebral palsy
}

\author{
G. Marrè-Brunenghi $\cdot$ R. Camoriano $\cdot$ M. Valle $\cdot$ S. Boero
}

Received: 23 October 2007 / Accepted: 14 January 2008 / Published online: 17 March 2008

\begin{abstract}
Psoas muscle spasticity is hypothesised as a rare cause of low back pain in patients with infantile cerebral palsy. The authors describe a new manoeuvre for the study of psoas tenderness and ultrasound (US)-guided transabdominal botulinum toxin injection technique. A possible causal relationship between psoas tension and low back pain was found incidentally in two examined cases. In subsequent patients, botulinum toxin was injected and, in cases of disappearance of symptoms, the psoas tendon was sectioned at the pelvic brim with definitive disappearance of pain. The relationship between psoas tension and low back pain in patients with infantile cerebral palsy seems likely, given the result in the four patients.
\end{abstract}

Keywords Cerebral palsy · Low back pain · Botulinum toxin · Psoas muscle

\footnotetext{
G. Marrè-Brunenghi $(\bowtie) \cdot S$. Boero

Department of Orthopaedics

Giannina Gaslini Institute

Largo G. Gaslini 5

I-16147 Genoa, Italy

e-mail: giorgiomarre@ ospedale-gaslini.ge.it

R. Camoriano

Physical Therapy Unit

Giannina Gaslini Institute, Genoa, Italy

M. Valle

Radiology Service

Giannina Gaslini Institute, Genoa, Italy
}

\section{Introduction}

Clinically, patients with infantile cerebral palsy sometimes present with severe low back pain and a pressing request for reduction of painful symptoms. A causal relationship between low back pain and psoas spasticity is hypothesised. Effective spasticity treatment allows lowering of tension and thus an altered lumbosacral posture.

Anatomic and functional aspects

The iliopsoas muscle is composed of the psoas and iliacus muscle [1]. These two portions, which are clearly separated cranially, join together caudally and are both inserted in the lesser femoral trochanter. Interest is focused on the psoas muscle.

The psoas muscle is fusiform, elongated and extending from the lumbar region to the lesser trochanter. It is proximally inserted in the groove formed by vertebral bodies and their transverse processes (vertebral-costiform angle). Through a series of medially concave fibrous arches, its anterior fibres are inserted in the anterior and lateral surfaces of D12 to L5 intervertebral disks, while posterior fibres originate from the 12 th rib and from costiform processes of the first four lumbar vertebrae. The psoas muscle thus forms a fusiform muscular body with maximum thickness at the level of the sacroiliac joint. Then it passes through the lumbar region and the pelvis, emerging from this, below, through a groove on the anterior border of the iliac bone between the anterior-inferior iliac spine and the iliopectineal eminence. It is inserted in the posterior surface of the lesser trochanter through a strong tendon originating above, in the pelvis, on the muscle's posterior surface. 
Different nerves of the lumbar plexus pass through the psoas major and minor muscle, namely: ilioinguinal, femorocutaneous, genitofemoral, obturator and femoral.

The psoas muscle acts as flexor of the hip, also inducing external rotation and adduction. In the erect position, it acts as flexor of the trunk forward.

In patients with infantile cerebral palsy, especially in tetraparesis or paraparesis (diplegia), psoas muscle spasticity may cause hip flexion deformity and a hollow back, either dynamic or structured, i.e., caused by spastic contraction or fixed retraction of the muscle [2, 3]. After causing these deformities, muscle tension may cause pain in a still unknown way.

\section{Materials and methods}

Four patients (age range 6-21 years) with symptomatic low back pain and CP diplegia or tetraparesis are described.

These patients are part of research on botulinum toxin treatment approved by the ethical board of the Gaslini Institute, following the Declaration of Helsinki. All the patients and their parents were informed of the treatment and gave their written consent.

In the first two patients, only clinical observations of a "normal" treatment were made. These observations were used to treat the last two patients; they were included in the study for the presence of the following criteria: cerebral palsied patients; persistent low back pain, unresponsive to every treatment; positivity to the psoas tenderness manoeuvre.

Numerical Rating Scale (NRS) in the $0-10$ version [4] is used to measure pain preoperatively and in the follow-up.

\section{Thomas test}

This test is used to detect a hip flexion deformity and/or contracture [5]. The patient is supine with the legs free outside the bed. The examiner places one hand behind the small of the patient's back, between it and the couch. There is a small gap here due to normal lumbar lordosis. The examiner abolishes the lumbar lordosis by flexing one patient's hip and feels the lumbar spine flatten out onto his hand. When the lumbar spine is flat, if the patient's other (studied) hip is lifted off the bed, that is due to a deformity/contracture. The entity of the deformity/contracture is measured by the angle of hip flexion between the bed and the thigh.

\section{Staheli test}

The "prone hip extension test" is described by the author as a simple, convenient and reliable method of measur- ing hip flexion contracture in spastic patients [6]. The patient is prone with the lower limbs free outside the bed. One examiner's hand is on the sacrum to feel its horizontal position; the second examiner's hand lifts the lower limb of the studied hip until the sacrum begins to move up. The angle between the lifted lower limb and the horizontal plane is measured to quantify the hip flexion contracture.

Manoeuvre for the study of psoas tenderness

The patient is supine with hips and knees flexed at $90^{\circ}$, if necessary with the legs slightly supported by the examiner, and is asked to bend one hip against resistance (isometric contraction). The manoeuvre is positive if the patient then suffers low back pain.

Ultrasound (US)-guided transabdominal botulinum toxin injection technique for the psoas muscle

The patient is sedated and in the supine position (Fig. 1). US examination of the abdomen is performed with a 5-7.5 MHz linear probe (Hitachi, Tokyo, Japan), displacing the intestinal loops medially starting from the paramedian lower quadrants. Visualisation of vertebral bodies and major iliac vessels serve as a reference. Lateral to the spine, the body of the psoas muscle is visualised and identified thanks to its movement, which is synchronous during hip flexion-extension. A needle of adequate length is used and botulinum toxin is diluted with at least $2 \mathrm{ml}$ of saline solution. The drug is injected in 4 sites, starting caudally or paraumbilically with US visualisation of liquid flow coming out of the needle.

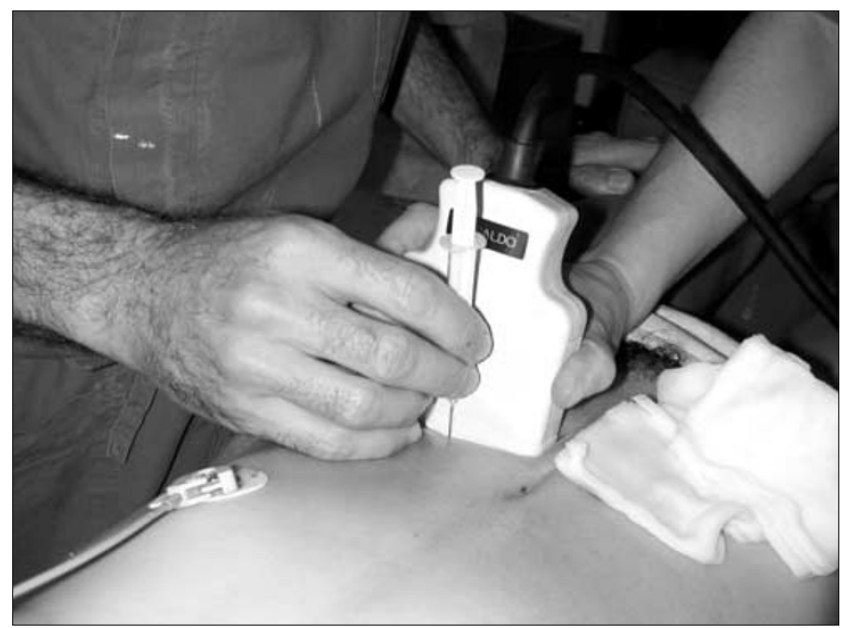

Fig. 1 US-guided transabdominal botulinum toxin injection technique for the psoas muscle 


\section{Case series}

This study was started on the basis of clinical observations of the patients described below.

\section{Case 1}

Case 1 was a male, aged six years, suffering from spastic tetraparesis. He walked with a rollator. The child was admitted to our unit because of frequent low back pain $(\mathrm{NRS}=6)$. Blood tests, X-ray of lumbar spine and neurophysiologic evaluation were performed and all results were normal. Repeated clinical examinations showed hypertonus of hamstrings, slight flexion of hips due to psoas tension $\left(10^{\circ}\right.$ at Thomas manoeuvre and $0^{\circ}$ at Staheli manoeuvre bilaterally) and adductor spasticity. Botulinum toxin (Botox, Allergan Pharmaceuticals, Westport, Ireland) was injected into the hamstrings and hip adductors (50 $\mathrm{U}$ for each muscle per $17 \mathrm{~kg}$ of body weight). At 45 days postinjection, his hip flexion deformity had decreased ( $<5^{\circ}$ at Thomas manoeuvre) and, most of all, there was complete regression of pain $(\mathrm{NRS}=0)$. This had not reappeared 2 years after our drug administration.

Psoas relaxation and pain regression were not immediately related, as botulinum toxin was injected into several other muscles. However, since pelvic posture change was clearly observed, a relationship between botulinum injection, iliopsoas relaxation and disappearance of pain was later hypothesised. The other cases reported here seem to confirm this assumption.

\section{Case 2}

Case 2 was male, aged 18 years, with spastic diplegia with considerable right prevalence. The right hip was flexed $\left(10^{\circ}\right.$ on the right side during Thomas and Staheli manoeuvres) and adducted. The right knee was flexed $\left(-15^{\circ}\right)$ and the right foot presented equinus deformity $\left(-5^{\circ}\right)$. After accurate orthopaedic and physiatric evaluation assisted by gait analysis, bilateral psoas release at the pelvic brim [7, 8] and correction of equinus deformity (according to Baumann [9]) were performed. In the postoperative period, the hip and foot deformities disappeared and after adequate physiotherapy the gait improved considerably. Low back pain (NRS $=5$, referred after surgery), which had been present for about two years and had not been mentioned by the patient before surgery, regressed completely $(\mathrm{NRS}=0)$. This was such a change for this patient and the parents that the result was described to us as 'a major change'.

The two cases described above focused the attention of the clinicians on the association between psoas tension and low back pain with regression of pain after surgeryor even drug-induced muscle relaxation.

\section{Case 3}

Case 3, a 12-year-old girl, suffered from spastic tetraparesis. She was only able to walk at home using a rollator. The girl had already been operated on in another institution at 7 years of age with bilateral myofascial lengthening of hamstrings and transfer of rectus femoris to semitendinosus. Notwithstanding her obvious hip flexion, psoas release at the pelvic brim had not been performed as it was considered contraindicated in the overall therapeutic programme. The girl was admitted to the unit at 10 years of age for severe low back pain $(\mathrm{NRS}=7$ ) preventing her from obtaining regular rest or effective physiotherapy and occasionally even walking. At that time she was diagnosed as suffering from an inflammatory disease affecting the sacroiliac joints. Her pain did not disappear in spite of administration of adequate anti-inflammatory therapy, and was characterised by alternating regression and acute phases, but generally presented a worsening trend. At 12 years of age, the girl was readmitted to the unit because of very severe back pain (NRS $=9$ ) preventing rest and almost completely preventing walking and standing.

Examination showed bilateral $20^{\circ}$ hip flexion (both with Thomas and Staheli manoeuvres). Botulinum toxin (Botox, Allergan Pharmaceuticals, Westport, Ireland) at high dosage (200 U per side per $42 \mathrm{~kg}$ of body weight) was injected to the right and left psoas muscles. On the following day, the girl reported complete disappearance of her low back pain (NRS =1).

After 15 days, when favourable results from her botulinum toxin injections were expected, pain reappeared with the same intensity (NRS $=8$ ).

Therefore bilateral psoas muscle release at the pelvic brim [7, 8] was performed. At postoperative visits after 1.5, 6 and 12 months, respectively, the girl reported no pain $(\mathrm{NRS}=0)$ and, after a period of initial instability, some gait improvement. Clinical observation showed improvement of hip flexion $\left(10^{\circ}\right.$ bilaterally on Thomas and Staheli manoeuvres).

\section{Case 4}

Case 4 was a 21-year-old man suffering from a spasticdystonic tetraparesis, but with an autonomous gait. The patient was admitted to the unit for severe low back pain (NRS $=7$ ) requiring anti-inflammatory drug treatment, even by intravenous injections. This produced a poor result 
$(\mathrm{NRS}=5)$. Many clinical and investigative examinations had been performed in another institution with negative results. The first examination showed hip flexion $\left(20^{\circ}\right.$ at Thomas manoeuvre and $10^{\circ}$ at Staheli manoeuvre bilaterally), and tenderness from the psoas tension (elicited by the hip examination/manoeuvre described above). His gait pattern showed a hollow back due to pelvic anteversion. Pain reduced the patient's ability to walk and to keep the erect position. Botulinum toxin in right and left psoas with the technique already described was injected. In this patient, the drug administered (Botox, Allergan Pharmaceuticals, Westport, Ireland, $200 \mathrm{U}$ per side per $68 \mathrm{~kg}$ of body weight) had a classic effect: after 15 days, the patient had complete regression of pain $(\mathrm{NRS}=0)$ and was well for 3 months. Then pain reappeared with the same intensity as before (NRS $=7$ ). Surgical psoas release was decided; this brought about complete disappearance of painful symptoms (NRS $=0)$ and decreased hip flexion $\left(10^{\circ}\right.$ at Thomas manoeuvre and $5^{\circ}$ at Staheli manoeuvre bilaterally). Pain was absent even 12 months later.

\section{Discussion}

Muscle visualisation with the US technique described above is optimal and botulinum injection in the correct muscle portal is certain, which makes monitoring with a myostimulator as suggested by Willenborg et al. [10] unnecessary. In our clinical practice in children, posterior access to the muscle is not feasible with CT monitoring [11] because of concerns about exposure to radiation. Techniques without instrumental monitoring [12] may fail because of uncertain injection site access. An inguinal injection route to the psoas muscle $[13,14]$ does not meet our therapeutic needs as, through this portal, the drug is injected only into the iliac muscle, as, at this level, the psoas muscle is already tendinous.

Despite several examinations, in this series other explanations of the patients' low back pain were not found. Particularly spasm of paraspinal muscles [15], signs of dystonia [16], other neurological involvement (central or peripheral), intrapelvic diseases or orthopaedic problems (trauma, disk protrusion) were not found. The origin of the pain from psoas muscle was only a clinical observation in the first two cases. Only the other two really follow the presented theory. This is, with the small number of cases, a limit for this series.

In the literature there are no publications showing a relationship between psoas muscle and back pain in patients with infantile cerebral palsy.

Though based on few patients, the theory correlating psoas tension to low back pain seems to be validated by the four cases described above.
In order to evaluate any possible iliopsoas role, patients affected by cerebral palsy and low back pain should be checked for the following features:

1. presence of continuing and severe (minimum NRS =5) low back pain in patients with infantile cerebral palsy in whom any other spinal or intrapelvic diseases have been excluded;

2. presence of hip flexion (positivity of Thomas and/or Staheli manoeuvres);

3. positive manoeuvre for the study of psoas tenderness;

4. regression of pain after US-guided transabdominal injection of botulinum toxin in the psoas muscle.

Only when these requirements are met, it is possible to propose psoas tendon release at the pelvic brim $[7,8]$ to the patient. The limit of this study, which is still in its initial phase, is the small number of patients and the short followup. These preliminary data, which can be considered based only on clinical impressions, need to be confirmed by a larger number of cases and a longer follow-up.

Acknowledgements The authors of this article want to thank Mr J.H. Patrick MD. His precious help in studying cerebral palsy made it possible for the authors to direct their attention to the small changes they have seen in the first patients and the consequent results in the others. The authors thank also Mr J.H. Patrick for his supervision in the revision of the article.

Conflict of interest statement The authors declare that they have no conflict of interest related to the publication of this manuscript.

\section{References}

1. Testut L, Latarjet A (1972) Il muscolo ileopsoas. In: Testut L, Latarjet A (eds) Trattato di Anatomia Umana, Vol II. UTET, Turin, pp 237-244

2. Delp SE, Arnold AS, Speers RA et al (1996) Hamstrings and psoas lengths during normal and crouch gait: implications for muscle-tendon surgery. J Orthop Res 14:144-151

3. DeLuca PA, Ounpuu S, Davis RB et al (1998) Effects of hamstrings and psoas lengthening on pelvic tilt in patients with spastic diplegic cerebral palsy. J Pediatr Orthop 18:712-718

4. McGuire DB (1984) The measurement of clinical pain. Nurs Res 33:152-156

5. Andreini G, Mangiaracina A (1975) Semeiotica Ortopedica. Pozzi, Roma, pp 291-292

6. Staheli L (1977) The prone hip extension test: a method of measuring hip flexion deformity. Clin Orthop 123:12-15

7. Patrick JH (1996) Technique of psoas tenotomy and rectus femoris transfer: "new" operations for cerebral palsy diplegia - a description. J Pediatr Orthop B 5:242-246

8. Sutherland DH, Zilberfarb JL, Kaufman KR et al (1997) Psoas release at the pelvic brim in ambulatory patients with cerebral palsy: operative technique and functional outcome. J Pediatr Orthop 17:563-570

9. Baumann JU (1986) Treatment of paediatric spastic foot deformities. Orthopade 15:191-198

10. Willenborg MJ, Shilt JS, Smith BP et al (2002) Technique for iliopsoas ultrasound-guided active electromyography-directed 
botulinum A toxin injection in cerebral palsy. J Pediatr Orthop 22:165-168

11. Porta M (2000) A comparative trial of botulinum toxin type A and methylprednisolone for the treatment of myofascial pain syndrome and pain from chronic muscle spasm. Pain $85: 101-105$

12. Ward AB (1999) Botulinum toxin type A treatment of hip and thigh spasticity: a technique for injection of psoas major muscle. Eur J Neurol 6[Suppl 4]:S91-S93

13. Berweck S, Heinen F (2003) Recent advances. In: Berweck S, Heinen F, Urban J (eds) Treatment of cerebral palsy with botuli- num toxin. Principles, clinical practice, atlas. Child \& Brain, Bonn, Berlin, pp 137-142

14. Westhoff B, Seller K, Wild A et al (2003) Ultrasound-guided botulinum toxin injection technique for the iliopsoas muscle. Dev Med Child Neurol 45:829-832

15. Gallien P, Nicolas B, Petrilli S et al (2004) Role for botulinum toxin in back pain treatment in adults with cerebral palsy: report of a case. J Bone Spine 71:76-78

16. Comella CL, Schanon KM, Jaglin J (1998) Extensor truncal dystonia: successful treatment with botulinum toxin injections. Mov Disord 13:552-555 